\title{
Consumo de alcohol y sus efectos sobre la salud
}

GIORGIO SOLIMANO(1)

\section{INTRODUCCIÓN}

El consumo de alcohol en diferentes formas es una práctica muy extendida en el mundo y que se remonta a tiempos inmemoriales. En este contexto, hay que distinguir entre el consumo moderado, que es socialmente aceptado y no origina problemas de salud u otras consecuencias indeseables, y el consumo en exceso, que constituye un problema social de grandes proporciones a nivel mundial, con efectos devastadores sobre las condiciones de vida y de salud de los individuos y de grupos importantes de población.

La mayoría de los países y diferentes organismos internacionales han tomado medidas para prevenir el consumo excesivo de bebidas alcohólicas y sus efectos, las que se traducen en leyes y diferentes normativas que están en permanente revisión. Nuestro país es uno de ellos, donde actualmente se debate sobre el perfeccionamiento de la legislación existente.

\section{SITUACIÓN MUNDIAL}

La OMS estima que a nivel mundial, alrededor de 2 billones de personas consumen bebidas alcohólicas y 76,3 millones sufren trastornos relacionados con el consumo de alcohol. En términos globales, existe una relación causal entre consumo de alcohol y más de 60 tipos de enfermedades y traumatismos.

Según la OMS, el alcohol causó el año 2002
1,8 millones de muertes - 3,2\% del total - y la pérdida de 58,3 millones, 4\% del total, de los DALY (Disability Adjusted Life Year) (OMS, 2002). Los accidentes y traumatismos no intencionales fueron responsables de un tercio de esas muertes y las afecciones neurosiquiátricas del $40 \%$ de los 58,3 millones de DALY.

Teniendo en cuenta la gravedad del problema, la OMS, a partir de 1996, ha desarrollado a nivel mundial una Base Global de Datos con el propósito de proveer información estandarizada sobre la evolución de la situación y de los problemas relacionados con el consumo de alcohol. El más reciente corresponde al Informe sobre la salud en el mundo, año 2004.

Según este informe, el consumo de alcohol puro en Chile era de 6,02 litros per cápita, mayor que en Brasil y Colombia e inferior a Argentina, donde alcanzaba a 8,55 litros per cápita. Por su parte, en Alemania, Francia y Hungría éste oscilaba entre los 12 y 14 litros.

Finalmente, los Estados Miembros de OMS han aprobado varias resoluciones llamando a implementar estrategias y programas eficaces para reducir las consecuencias sanitarias y sociales del uso nocivo de alcohol, al igual que han hecho notar la imperiosa necesidad de promover la movilización y la participación activa de todos los grupos sociales y económicos interesados a nivel nacional.

\footnotetext{
(1) Escuela de Salud Pública, Facultad de Medicina, Universidad de Chile. gsolimano@med.uchile.cl
} 


\section{SITUACIÓN EN CHILE}

En nuestro país, el consumo excesivo de alcohol es uno de los principales problemas de salud que afectan a la población. Al año mueren 7.600 personas por causas asociadas a su ingesta; casi un millón de chilenos es "bebedor problema" y sus edades fluctúan principalmente entre los 15 y los 35 años (Figura 1).

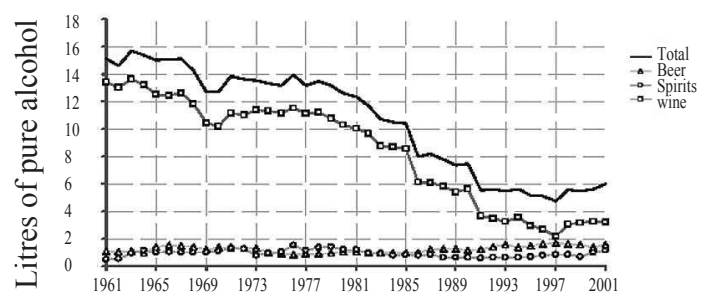

Sources: FAO (Food Agriculture Organization of the United Nation), World Drink Trends 2003

Figura 1. Chile: Registro de consumo percápita de adultos (15 años y más).

La situación es particularmente alarmante entre los adolescentes. Chile es uno de los países de América Latina con más alto índice de consumo de bebidas alcohólicas en este segmento, superando incluso a los Estados Unidos. Los escolares chilenos de octavo año básico, así como de segundo y cuarto medio, presentan la segunda tasa más alta de consumo reciente de alcohol $(62,7 \%)$ en Latinoamérica, luego de Uruguay $(67,2 \%)$. El ranking continúa con Ecuador $(42,2 \%)$, Nicaragua $(37,7 \%)$ y Guatemala (30,6\%).

Se estima que unos 400 mil niños de entre 9 y 15 años toman licores a diario y 200 mil jóvenes ya son alcohólicos. Asimismo, se sabe que el alcohol es la principal puerta de entrada a las drogas: el 90\% de los jóvenes calificados como bebedores problema, están al mismo tiempo consumiendo algún estupefaciente (Figura 2).

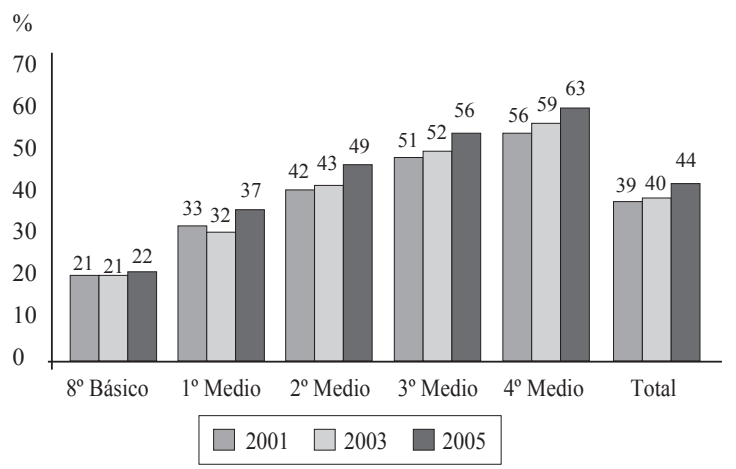

Fuente: CONACE. Sexto estudio nacional de drigas en población escolar de Chile, 2005.

Figura 2. Evolución de prevalencia de último mes de consumo de alcohol, por cursos.

\section{EFECTOS DEL CONSUMO DE ALCOHOL EN CHILE}

La información que presentan las siguientes figuras muestran los efectos del consumo de alcohol en nuestro país y su impacto sobre la morbilidad y mortalidad generales por causas específicas (Figuras 3 a 6).

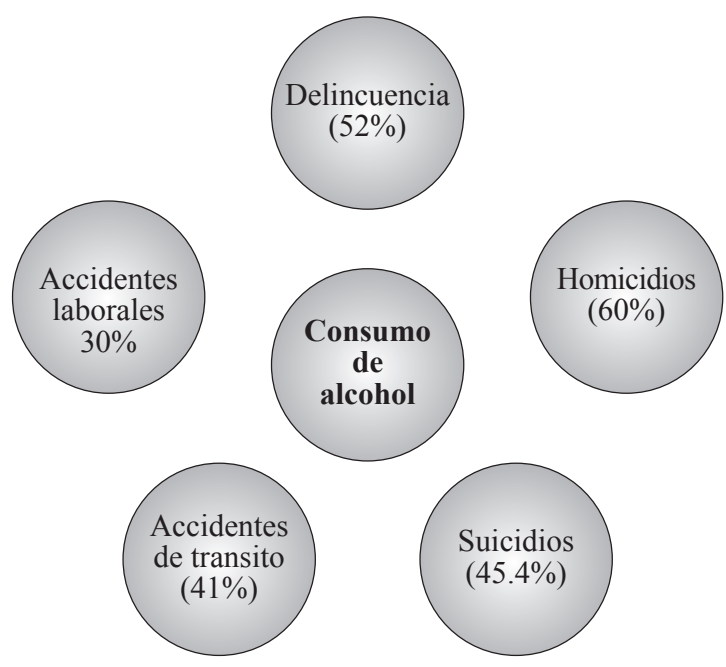

Figura 3. Efectos del consumo de alcohol en Chile. 


\begin{tabular}{|c|c|c|c|c|}
\hline CIE - 10 & Causas de defunción & Defunciónes & Tasa* & $\%$ \\
\hline $\mathrm{I} 20-\mathrm{I} 25$ & Enfermedades isquémicas del corazón & 7.967 & 49,5 & 9,2 \\
\hline I60-I69 & Enfermedades cerebrovasculares & 7.783 & 48,4 & 9,0 \\
\hline K70-K76 & Cirriosis y otras enfermedades del hígado & 4.177 & 26,0 & 4,8 \\
\hline $\mathrm{I} 30-\mathrm{I} 52$ & Otras formas de enfermedades del corazón & 3.762 & 23,4 & 4,4 \\
\hline E10-E14 & Diabetes mellitus & 3.415 & 21,2 & 4,0 \\
\hline J12-J18 & Neumonía & 3.415 & 21,2 & 4,0 \\
\hline $\mathrm{I} 10-\mathrm{I} 13$ & Enfermedades hipertensivas & 3.132 & 19,5 & 3,6 \\
\hline $\mathrm{C} 16$ & Tumor maligno del estómago & 3.052 & 19,0 & 3,5 \\
\hline J40-J44 & $\begin{array}{l}\text { Bronquitis, enfisema y otras enfermedades } \\
\text { pulmonares obstructivas crónicas }\end{array}$ & 2.835 & 17,6 & 3,3 \\
\hline \multirow[t]{4}{*}{ C33-C34 } & $\begin{array}{l}\text { Tumor maligno de la traquea, de los bronquios } \\
\text { y del pulmón }\end{array}$ & 2.222 & 13,8 & 2,6 \\
\hline & Acumulado principales causas & 41.760 & 259,5 & 48,5 \\
\hline & Las demás causas & 44.378 & 276,8 & 51,5 \\
\hline & TOTAL & 86.138 & 535,2 & 100,0 \\
\hline
\end{tabular}

\begin{tabular}{|c|c|c|c|c|c|c|}
\hline \multirow[b]{2}{*}{ año } & \multicolumn{2}{|c|}{ Ambos sexos } & \multicolumn{2}{|c|}{ Hombres } & \multicolumn{2}{|c|}{ Mujeres } \\
\hline & Defunciónes & $\begin{array}{c}\text { Tasa } \\
*\end{array}$ & Defunciónes & $\begin{array}{c}\text { Tasa } \\
*\end{array}$ & Defunciónes & $\begin{array}{c}\text { Tasa } \\
* \\
\end{array}$ \\
\hline 1990 & 4.075 & 30,9 & 2.949 & 45,3 & 1.126 & 16,9 \\
\hline 1991 & 3.231 & 24,1 & 2.291 & 34,5 & 940 & 13,8 \\
\hline 1992 & 2.718 & 19,9 & 1.992 & 27,9 & 836 & 12,1 \\
\hline 1993 & 2.764 & 23,2 & 1.921 & 27,9 & 843 & 12,0 \\
\hline 1994 & 3.286 & 25,7 & 2.239 & 32,0 & 1.047 & 14,6 \\
\hline 1995 & 3.699 & 26,9 & 2.636 & 37,0 & 1.063 & 14,6 \\
\hline 1996 & 3.929 & 25,0 & 2.837 & 39,3 & 1.092 & 14,8 \\
\hline 1997 & 3.651 & 24,1 & 2.684 & 37,2 & 967 & 13,1 \\
\hline 1998 & 3.611 & 21,8 & 2.582 & 34,8 & 1.029 & 13,6 \\
\hline 1999 & 3.320 & 22,8 & 2.348 & 31,2 & 972 & 12,7 \\
\hline 2000 & 3.504 & 25,4 & 2.484 & 32,6 & 1.020 & 13,1 \\
\hline 2001 & 3.954 & 25,4 & 2.854 & 37,0 & 1.100 & 14,0 \\
\hline 2002 & 3.997 & 26,1 & 2.847 & 36,5 & 1.150 & 14,5 \\
\hline 2003 & 4.154 & 26,1 & 2.976 & 37,8 & 1.179 & 14,7 \\
\hline 2004 & 4.177 & 26,0 & 2.960 & 37,2 & 1.217 & 15,0 \\
\hline
\end{tabular}

CIE - 9(hasta 1996): 570- 573; 576.1

CIE - 10 (desde 1997): K70- K76

Tasa por cien mil habitantes

Fuente: DEIS. http://deis.minsal.cl/index.asp

Figura 4. Mortalidad para ambos sexos, según las principales causas específicas de defunción. Chile, 2004. 


\section{LA REALIDAD EN EL 2005}

En el último año de análisis, los siniestros de tránsito en los cuales se asoció como causa basal el consumo de alcohol en los conductores alcanzaron, la no despresiable suma de 2.927 sucesos, dejando como consecuancia la pérdida de 209 vidas humanas y un total de 4.062 lesionados. En relación a las ponderaciones de tipo porcentual, los siniestros por esta causa abarcaron el 6,32\% del total, los fallecidos por esta razón sumaron el 12,85\%, mientras que el 8,50\% de los lesionados se concentró en estos siniestros.

Ranking de siniestros de tránsito según causas de ocurrencia (2005)

\begin{tabular}{|l|r|r|r|r|r|r|}
\hline \multirow{2}{*}{ Causa del siniestro } & \multirow{2}{*}{ Siniestros } & \multirow{2}{*}{ Fallecidos } & \multicolumn{3}{|c|}{ Lesionados } & \multirow{2}{*}{ Total de lesionados } \\
\cline { 4 - 7 } & & & Gaves & Menos graves & Leves & 18.396 \\
\hline Imprudencia del conductor & 22.765 & 311 & 2.134 & 1.651 & 14.611 & 7.512 \\
\hline Desobediencia a señalización & 6.273 & 70 & 589 & 658 & 6.265 & 6.612 \\
\hline Pérdida de control & 4.914 & 210 & 1.093 & 678 & 4.841 & 3.874 \\
\hline Imprudencia de peatón & 3.929 & 378 & 1.040 & 501 & 2.333 & $\mathbf{4 . 0 6 2}$ \\
\hline Alcohol en conductor & $\mathbf{2 . 9 2 7}$ & $\mathbf{2 0 9}$ & $\mathbf{7 0 3}$ & $\mathbf{5 0 9}$ & $\mathbf{2 . 8 5 0}$ & 2.318 \\
\hline Exeso de velocidad & 1.514 & 137 & 411 & 304 & 1.603 & 940 \\
\hline Imprudencia de pasajero & 920 & 12 & 139 & 113 & 688 & 1.574 \\
\hline Fallas mecánicas & 819 & 22 & 202 & 153 & 1.219 & 527 \\
\hline Alcohol en peatón & 642 & 156 & 209 & 63 & 255 & 481 \\
\hline Otras causas & 505 & 53 & 55 & 57 & 369 & 378 \\
\hline Causas no determinadas & 502 & 1 & 59 & 44 & 275 & 824 \\
\hline Drogas y/o fatiga de conductor & 378 & 59 & 168 & 117 & 539 & 253 \\
\hline Deficiencias viales & 208 & 7 & 35 & 18 & 200 & 41 \\
\hline Alcohol en pasajero & 42 & 1 & 7 & 12 & 22 & $\mathbf{4 6 . 0 7 0}$ \\
\hline Totales & $\mathbf{4 6 . 3 2 8}$ & $\mathbf{1 . 6 2 6}$ & $\mathbf{6 . 8 4 4}$ & $\mathbf{4 . 8 7 8}$ & $\mathbf{4 7 . 7 9 2}$ \\
\hline
\end{tabular}

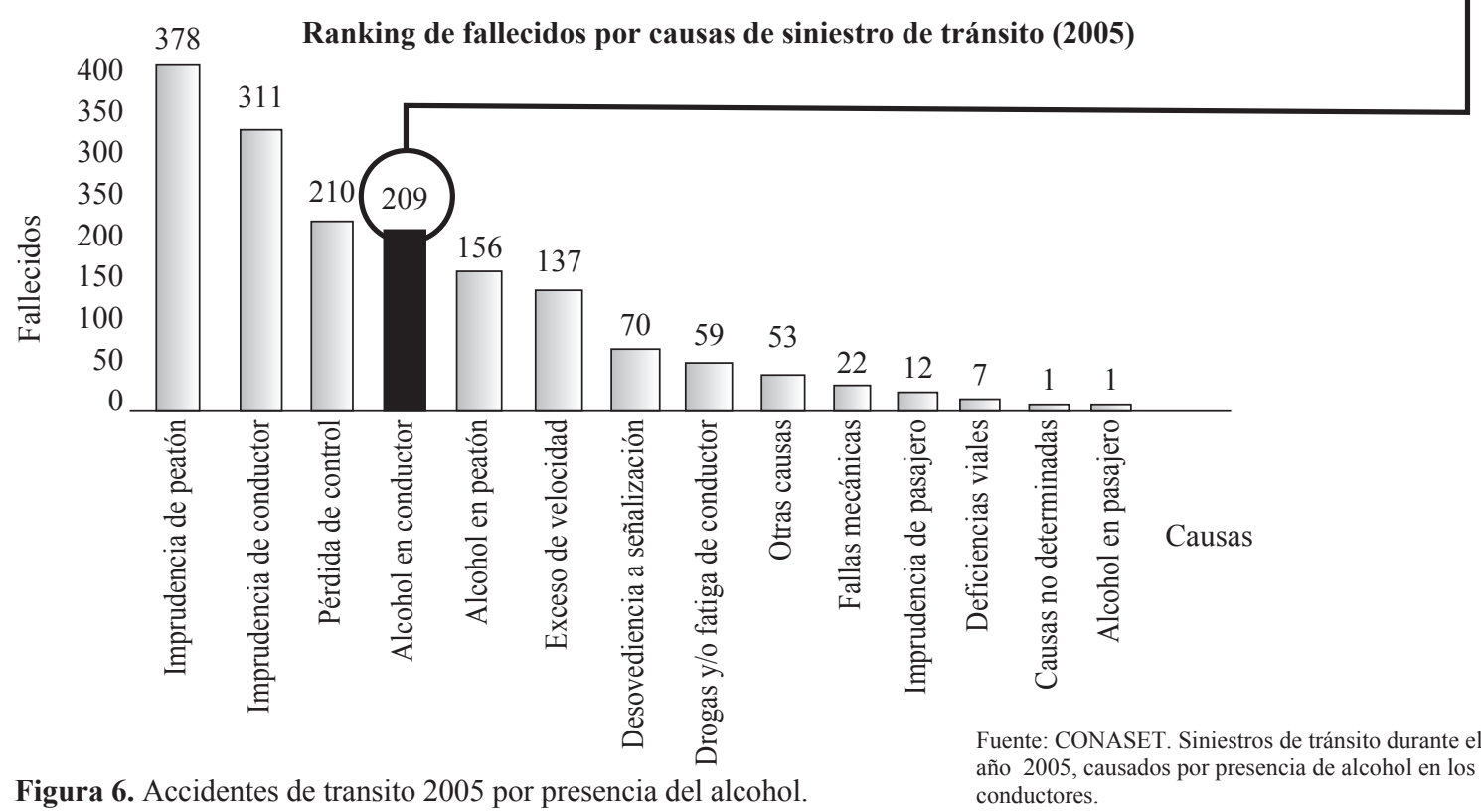




\section{LEGISLACIÓN}

En enero de 2004 se promulgó la ley 19.925 sobre Expendio y Consumo de Bebidas Alcohólicas, que se adecua a los nuevos estilos de vida y patrones de consumo de los chilenos. Esta ley introdujo cambios importantes, como la obligación de impartir programas para estimular hábitos de vida saludables en todos los establecimientos de educación; la prohibición de expender bebidas alcohólicas a menores de edad en cualquier establecimiento, el ingreso de menores de 18 años a los bares, tabernas y cabarets y la prohibición del ingreso de menores de 16 años a discotecas.

En la actualidad, se discuten nuevas modificaciones a la legislación existente, con el fin de corregir insuficiencias y hacerlas más estricta, especialmente en las medidas que dicen relación con el consumo de alcohol en los jóvenes.

Entre las medidas propuestas, destacan la prohibición del ingreso de menores de 18 años a discotecas o salones de baile; la obligación de los establecimientos que expendan bebidas alcohólicas a exigir carné de identidad, o cualquier documento que acredite la edad, a aquellos compradores que tengan aparentemente menos de 18 años y la posibilidad de que los inspectores municipales puedan solicitar el carné de identidad a compradores.

También se modifican las disposiciones en el caso de menores de 18 años sorprendidos en la vía pública consumiendo bebidas alcohólicas o circulando bajo los efectos del alcohol y se traspasan causas por expendio de alcohol a menores de 18 años a los Juzgados de Policía Local. 